\title{
基于红外成像技术的填埋场裸膜缺陷检测研究
}

\author{
陈亚宇，张 卫，孙焕奕，黄晓松 \\ (河北工程大学 机械与装备工程学院, 河北 邯䣋 056038)
}

\begin{abstract}
摘要: 利用高密度聚氯乙烯膜（HDPE）缺陷区与完整区同时刻温差特性, 采用红外热成像技术对 高密度聚氯乙烯膜进行缺陷检测。在持续热源作用下, 对不同面积及形状的缺陷进行红外图像采集, 记录膜表面不同区域的温度, 分析不同位置温度及红外缺陷阴影区随时间的变化规律, 提取温度特 征曲线及缺陷最佳检测时间范围。实验结果表明: 缺陷区与完整区温度随时间变化趋势整体相同, 但在相同时刻存在温度差异且红外图像采集时间为 $10 \sim 20 \mathrm{~min}$ 内温差值最明显, 可视为缺陷最佳检 测时间域, 红外热像采集时间为 $13 \mathrm{~min}$ 时, 红外图像边缘轮遊与实缺陷轮廊基本一致, 该时间为最 佳检测时间点。
\end{abstract}

关键词: 高密度聚氯乙烯膜; 红外热成像; 同步温差; 时间域

中图分类号：TN219 文献标识码：A 文章编号：1001-8891(2020)06-0598-05

\section{Defect Detection of Landfill Bare Film Based on Infrared Imaging Technology}

\author{
CHEN Yayu, ZHAGN Wei, SUN Huanyi, HUANG Xiaosong \\ (College of Mechanical and Equipment Engineering, Hebei University of Engineering, Handan 056038, China)
}

\begin{abstract}
Based on the simultaneous temperature difference of the HDPE film defect area and the intact area, defects of HDPE film were detected with infrared thermal imaging technology. Under the action of a continuous heat source: i) infrared images are collected for defects of different areas and shapes, ii) the temperature of different areas of the film surface is recorded, iii) the temperature changes of different locations and the shadow area of infrared defects with time are analyzed, and iv) finally, the temperature characteristic curve and the best defect detection time limit were studied. The experimental results show that the temperature trends of the defect and complete area are the same the whole time; however, there is a synchronous temperature difference, and the infrared temperature image acquisition time is clearly in the 10-20 mins. It can be regarded as the best detection time domain of the defect. When the thermal image acquisition time is 13 mins, the infrared image edge contour is the same as the real defect contour; therefore, it is the optimal detection time point.
\end{abstract}

Key words: HDPE film, infrared thermalimaging, sync temperature difference, time domain

\section{0 引言}

随着近年国民生活质量提高, 生活节奏加快, 随 之产生的各种垃圾显著增多 ${ }^{[1]}$ 。如今垃圾分类正如火 如茶进行 ${ }^{[2-3]}$, 但分类后的垃圾仍旧有很大一部分要 进行填埋处理。其中高密度聚氯乙烯膜 (HDPE) 作 为垃圾填埋场主要防渗材料, 其具有化学稳定性好、 渗透性小、抗老化性能优异、使用周期长等优势 ${ }^{[4-5]}$, 对防止垃圾带来次生污染起着至关重要的作用。
HDPE 膜铺设作为填埋场施工中最为重要的一个环 节, 但膜与膜间焊接时经常会出现虚焊、脱焊、漏焊 以及施工对膜平面造成的破损等情况, 都会为填埋场 将来使用埋下渗漏隐患, 因此需要重点管理与检测。 为实现垃圾安全填埋, 在填埋场投入使用之前及时发 现土工膜缺陷并进行补救具有非常重要的意义。

在现有垃圾填埋场裸膜缺陷检测方法中, 针对焊 缝主要采用压力检测, 即在双焊缝之间充气, 使双缝 之间压力达到规定值, 然后观察 1 5 min, 压力值无 
明显变化, 说明焊缝牢固, 符合要求; 若压力值变化 明显, 则焊缝之间存在缺陷 ${ }^{[6]}$ 。该方法原理简单且能 对缺陷进行判定, 但不能确定具体缺陷位置, 且在充 压时, 充压值控制不当易对 HDPE 膜造成损伤, 对 填埋场安全构成潜在威胁。针对 HDPE 膜平面缺陷, 主要采用电学检测方法 ${ }^{[7]}$, 即预先在膜上喷射水流, 膜下铺设检测电极, 通过给场内外电极供电, 由渗漏 检测仪对检测电极扫描来分析破损位置。该方法对裸 膜缺陷检测有良好检测效果, 但整套系统结构复杂而 且需要预先埋设, 使填埋场成本增加。因此本文提出 了一种基于红外成像技术的填埋场裸膜缺陷检测方 法。红外成像检测技术与前述检测方法相比, 其优势 在于不需要对 HDPE 材料进行直接接触, 避免了对 材料的接触伤害。其检测结果的可视性好, 能精准地 观测到缺陷位置及大小。

本文主要对 HDPE 膜缺陷区与完整区进行红外 热成像扫描 ${ }^{[8-10]}$, 对不同缺陷、不同时间段状况的 HDPE 进行实时红外热像采集, 根据缺陷区与正常区 的热对比度 ${ }^{[11-13]}$, 确定出最佳检测时间段。从而为 HDPE 膜缺陷的红外无损检测方法提供理论依据。

\section{1 检测原理}

\section{1 定位检测原理}

红外成像技术检测裸膜缺陷的原理为: 水流使 HDPE 膜缺陷区下土壤层湿润, 由于湿润土壤层和完 整区 HDPE 膜热扩散系数差异明显, 在持续热源作 用下, 缺陷区和完整区会出现同步温差特性, 通过红 外热像图来识别温差区域可以很好地检测到缺陷区 位置。

检测原理如图 1 所示, 首先由水流对 HDPE 膜 全表面进行降温处理, 在既定时间内由红外热像仪按 横纵既定采集路线对 HDPE 膜进行全方位采集。当 红外热像仪位于正常区时, 采集结果的热对比值无明
显变化, 当红外扫描仪位于缺陷区时, 扫描结果的热 对比值差异明显。

\section{2 缺陷检测模型建立}

热波在 HDPE 膜及土壤中传播时遵循热传导方 程, 为检测缺陷, 仅考虑深度方向上的热传导方式。 在导热系数确定情况下, 热传导方程式为:

$$
\nabla \cdot[k \nabla T(x, t)]-\rho c \frac{\partial T(x, t)}{\partial t}=-f(x, t)
$$

针对平面热源可得:

$$
f(x, t)=q \delta(x) \delta(t)
$$

式中: $\nabla$ 代表梯度; $f(x, t)$ 代表所处介质温度, $x$ 为所 处介质深度, $t$ 为时间; $q$ 为热流密度。

将式(2)带入式(1)得:

$$
\left\{\begin{array}{l}
\frac{\partial^{2} T(x, t)}{\partial x^{2}}-\frac{1}{\alpha} \frac{\partial T(x, t)}{\partial t}=-\frac{q}{k} \delta(x) \delta(t) \\
\alpha=k / \rho c v
\end{array}\right.
$$

式中: $\alpha$ 为所处介质热扩散系数; $k$ 为导热率; $\rho$ 为 密度; $c$ 为比热容; $v$ 为体积。当 $k$ 值越大时, 物体 对环境温度变化反应越快。如图 2 所示, 由破损区 湿润土壤作为导热介质, 其导热率 $k_{1}$ 最大, 对环境 温度变化反应最快。膜下湿润区由于受 HDPE 膜及 湿润土壤共同影响, 其等效 $k_{2}$ 值小于 $k_{1}$, 大于 $k_{3}$ 。 因此在酒水降温后一段时间内, 温度升势走向为 $T_{1}$ $<T_{2}<T_{3}$ 。

定义热相对值为 HDPE 膜正常区表面温度 $\left(T_{3}\right)$ 与缺陷区表面温度 $\left(T_{1}\right)$ 的差值, 用 $\Delta T$ 表示为:

$$
\Delta T=T_{3}-T_{1}
$$

通过分析上述公式可知, 由于水流作用, 当 HDPE 膜存在缺陷时, 将会对膜表面热场造成波动, 最终影响红外辐射检测过程。通过红外扫描仪采集红 外图像, 并在显示屏显示彩色热图, 从而可对 HDPE 膜缺陷进行检测和研究。

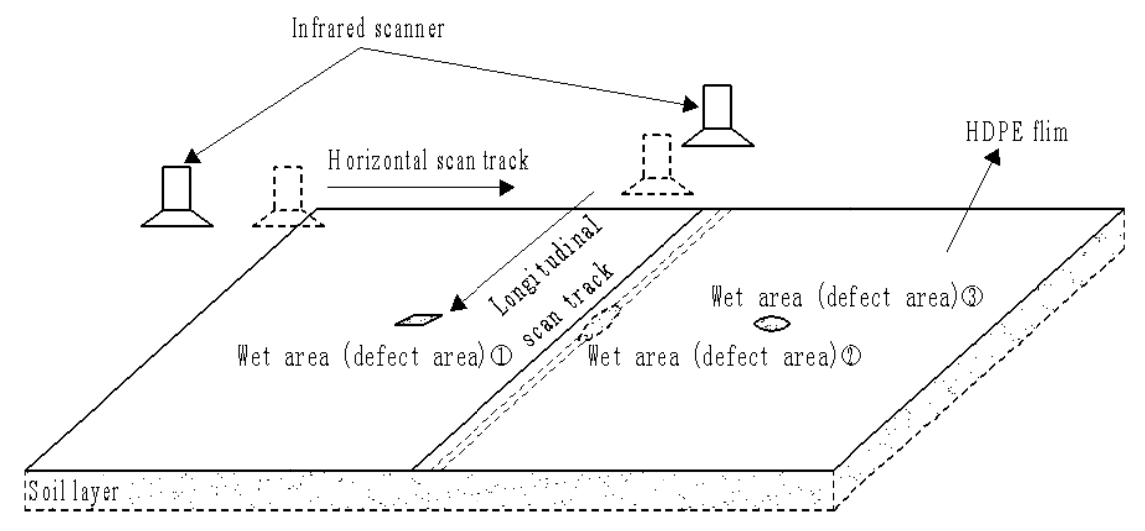

图 1 红外成像检测 HDPE 膜缺陷示意图

Fig.1 Infrared imaging detection of HDPE film defects 


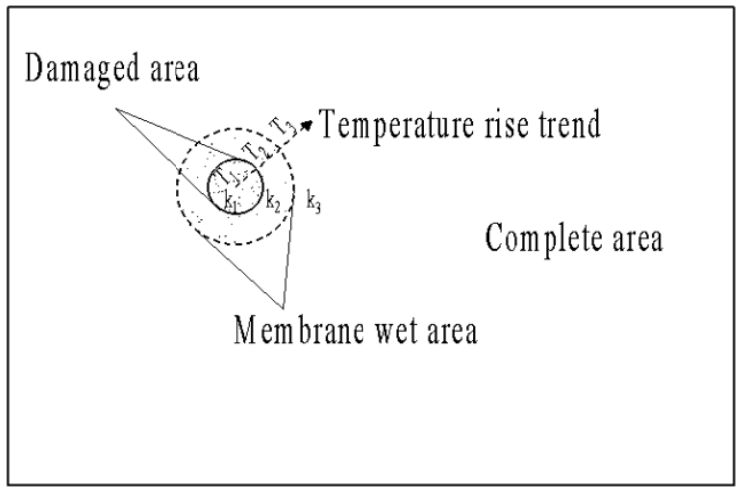

图 2 缺陷区热传导示意图

Fig.2 Schematic diagram of heat conduction in the defect area

\section{2 缺陷检测实验}

\section{1 实验材料及性能参数}

红外热像仪 (Fluke Tis10 9 Hz thermal imager), 精度为 $\pm 2^{\circ} \mathrm{C}$, 工作温度为 $-10^{\circ} \mathrm{C} \sim+50^{\circ} \mathrm{C}$, 分辨率 为 $80 \times 60$, 红外热像仪工作波段为 $7.5 \sim 14 \mu \mathrm{m}$, 满 足成像精度要求。HDPE 膜厚度为 $0.3 \mathrm{~cm}$, 穿刺强 度为 $249 \mathrm{~N}$, 断裂强度为 $53 \mathrm{~N}$, 导热率为 0.527 $\mathrm{W} /(\mathrm{m} \cdot \mathrm{K})$ 。湿润土层平均导热率 $1.052 \mathrm{~W} /(\mathrm{m} \cdot \mathrm{K})$ 。

\section{2 实验方法}

如图 3 所示, 制作尺寸为 $50 \mathrm{~cm} \times 35 \mathrm{~cm} \times 20 \mathrm{~cm}$ 的实验土箱, 最上层铺设 HDPE 膜, 膜上预置不同 类型缺陷, 缺陷区编号依次为 $1 、 2 、 3$, 其中编号 1 和编号 3 为不同形状的膜平面破损缺陷, 编号 2 为 双轨焊缝漏焊缺陷。膜下均匀铺设厚度为 $10 \mathrm{~cm}$ 的 土层。考虑到实况工作环境因素, 到实验结束之前 所有工作都在阳光充足条件下进行。
将暴晒 $30 \mathrm{~min}$ 后的 HDPE 膜铺设到土壤层上方 并采集红外热像图; 热像图采集完成后, 对 HDPE 膜进行喷水降温 (水温一般保持在 $26^{\circ} \mathrm{C}$ ), 然后对 缺陷区持续进行红外热像采集, 观测缺陷区与完整 区的红外热像温差变化, 记录具有明显差异的观测 值及时间点, 直至最终次采集结果无变化, 停止采 集实验; 将记录的图像进行对比分析找出最佳采集 时间域。

\section{3 实验结果分析}

对比图 4 中不同加热时间段红外图像可以看 出, 在对 HDPE 膜进行酒水降温之前, 膜表面温度 分布均匀保持在 $47^{\circ} \mathrm{C}$ 以上, 如图 4(a)所示。

$t=0.0 \mathrm{~min}$ 在刚经过水流降温的 HDPE 膜表面 温度整体降低, HDPE 膜缺陷区与完整区无明显差 异, 如图 4(b)所示。从 $t=0.5 \mathrm{~min}$ 开始, 缺陷区温 度开始出现差异, 水流由缺陷区渗漏到膜下土层中, 由于初始阶段水流与 HDPE 膜的直接接触面积大于 与缺陷区土层的接触面积, 最终导致土层的降温速 率明显小于膜的降温速率, 所以在图 4(c)中缺陷区 温度偏高。从 $t=10.0 \mathrm{~min}$ 开始, 由于土层的导热率 大于 HDPE 膜的导热率以及热源与缺陷区土层之间 存在对流, 导致 HDPE 膜完整区温度相对于缺陷区 温度更高, 如图 4(d)所示。 $t=11 \mathrm{~min}$, 在持续热源 作用下, 随时间加长, 缺陷区温度逐渐上升导致红 外模式下缺陷边缘轮廓持续减小。 $t=13 \mathrm{~min}$ 后, 缺 陷区温度随时间上升且接近 HDPE 膜完整区温度, 导致缺陷区边缘轮廓逐渐模糊, 如图 4(e)、(f)、(g) 所示。 $t=20 \mathrm{~min}$ 后缺陷区温度与完整区相近, 红外 边缘轮廓逐渐消失。如图 4(h)、(i)、(j) 所示。

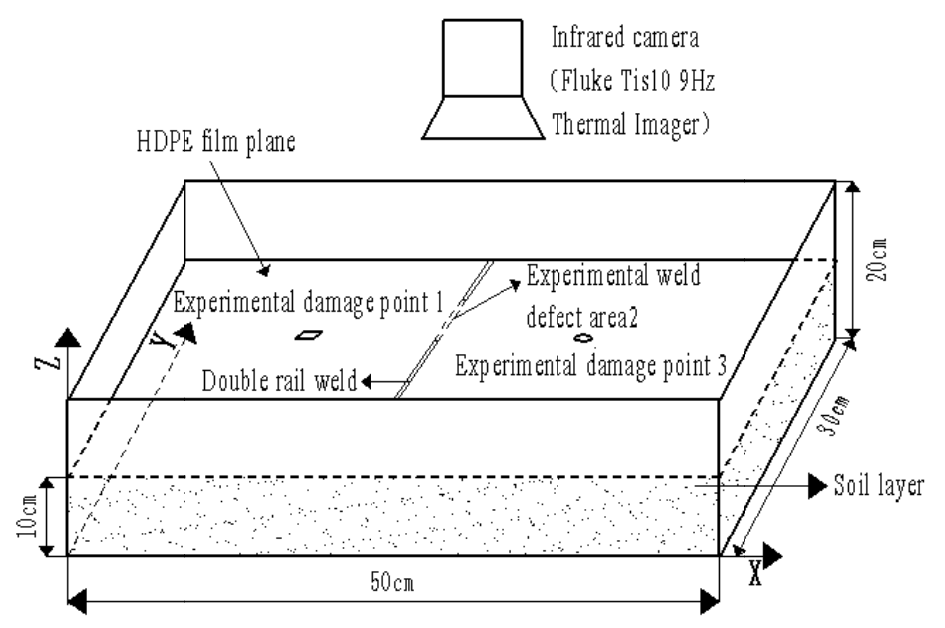

图 3 缺陷检测实验图

Fig.3 Defect detection experiment diagram 
为进一步分析 HDPE 膜缺陷状况, 提取了实验 缺陷区和正常区表面的温度曲线进行分析。缺陷区 和正常区表面温度曲线如图 5 所示, 由图 5 可知: 缺陷区与完整区温度变化趋势整体相同, 主要是由 于缺陷区湿润土层和 HDPE 膜完整区处在相同的作 用环境下; 但在 10 20 min 时相对温差值较大, 红
外热像仪能明显捕捉缺陷区位置, 主要是由于湿润 土层及 HDPE 膜材质不同, 导热率差异明显, 最终 会导致在相同时间段内出现温差值。同时由图 5 可 以进一步看出在 $t=13 \mathrm{~min}$ 时, 温差值最大, 观测 到的结果更加明显。

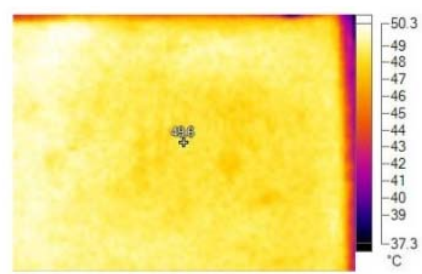

(a) 水流降温前

(a) Before the water cools down

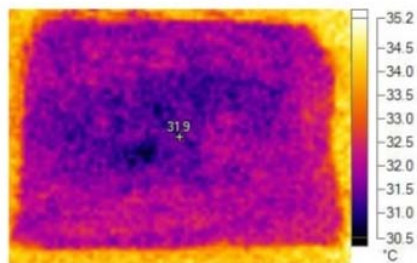

(b) 热源持续时间 $\mathrm{t}=0.0 \mathrm{~min}$ (b) Heat source duration $\mathrm{t}=0.0 \mathrm{~min}$

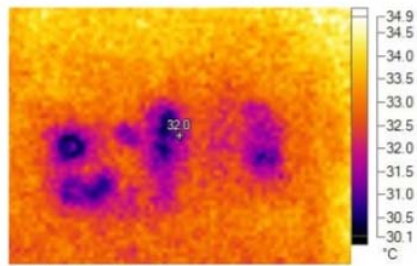

(e) 热源持续时间 $\mathrm{t}=11.0 \mathrm{~min}$

(e) Heat source duration $\mathrm{t}=11.0 \mathrm{~min}$

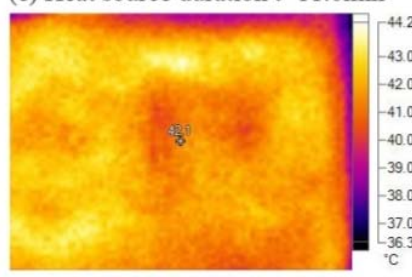

(h) 热源持续时间 $\mathrm{t}=20.0 \mathrm{~min}$

(h) Heat source duration $\mathrm{t}=20.0 \mathrm{~min}$

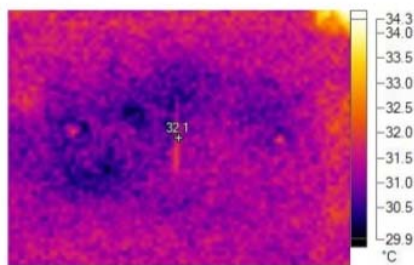

(c) 热源持续时间 $\mathrm{t}=0.5 \mathrm{~min}$ (c) Heat source duration $\mathrm{t}=0.5 \mathrm{~min}$

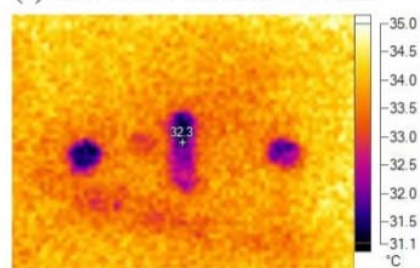

(f) 热源持续时间 $\mathrm{t}=12.0 \mathrm{~min}$

(f) Heat source duration $\mathrm{t}=12.0 \mathrm{~min}$

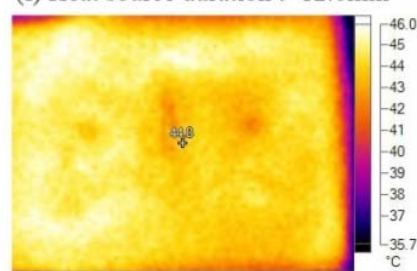

(i) 热源持续时间 $\mathrm{t}=21.0 \mathrm{~min}$

(i) Heat source duration $\mathrm{t}=21.0 \mathrm{~min}$

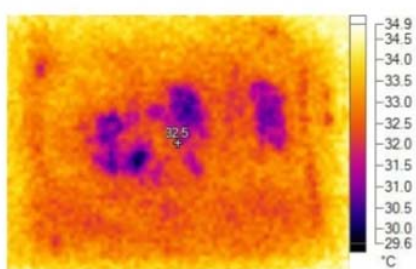

(d) 热源持续时间 $\mathrm{t}=10.0 \mathrm{~min}$

(d) Heat source duration $\mathrm{t}=10.0 \mathrm{~min}$

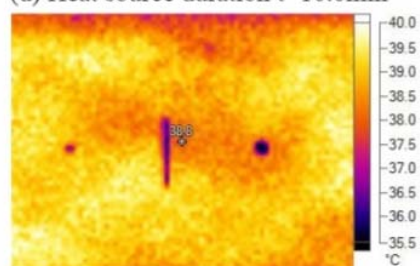

(g) 热源持续时间 $\mathrm{t}=13.0 \mathrm{~min}$

(g) Heat source duration $\mathrm{t}=13.0 \mathrm{~min}$

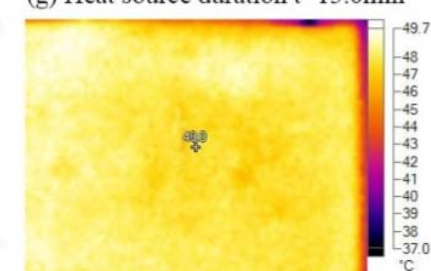

(j) 热源持续时间 $\mathrm{t}=25.0 \mathrm{~min}$

(j) Heat source duration $\mathrm{t}=25.0 \mathrm{~min}$

图 4 不同加热时间段热像图

Fig.4 Thermal image without heating period

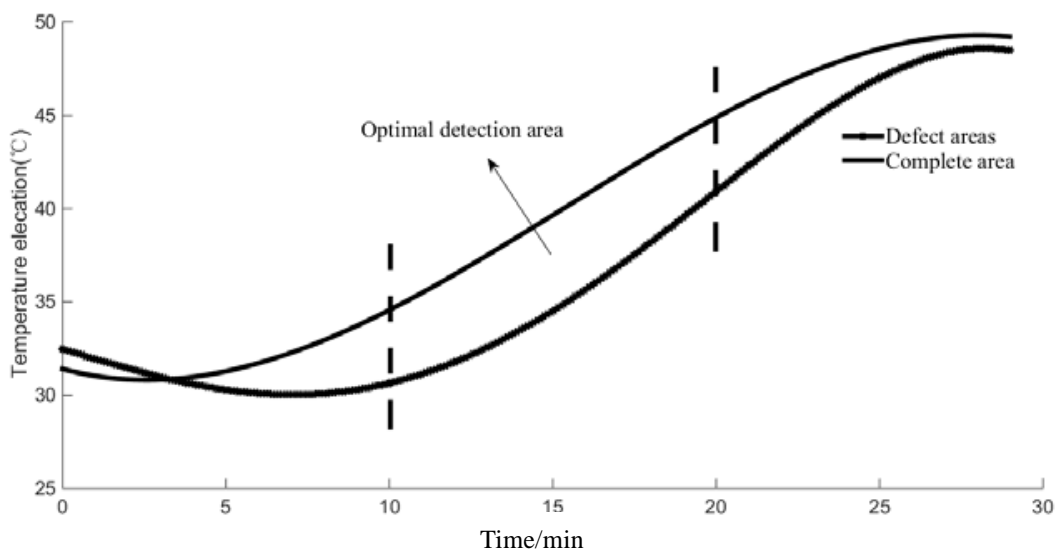

图 5 HDPE 膜缺陷区与完整区温度曲线对比图

Fig.5 HDPE film defect area and complete area temperature curve comparison chart 


\section{4 结论}

利用 HDPE 膜缺陷区与完整区的同时刻温差特 性, 采用红外成像技术对 HDPE 膜缺陷区进行检测 研究, 研究结果表明:

1）红外热像图中可以观测到缺陷区和完整区的 温度差异且在 $t=10 \sim 20 \mathrm{~min}$ 时, 温差特性最为显著, 该时间段为最佳检测时间域, 在 $t=13 \mathrm{~min}$ 时, $\Delta T=$ $4.5^{\circ} \mathrm{C}$, 该时间点为最大温差时间节点。

2) HDPE 膜在水流冷却及热源加热过程中, 膜 的缺陷区与完整区在相同时间段内会出现温差特性, 基于该特性利用红外热成像检测技术能够准确检测 到 HDPE 膜的缺陷位置。避免了对膜的接触式损坏, 实现了 HDPE 膜无损检测。同时也为 HDPE 膜材料 在不同场合的应用提供了一种全新的缺陷检测方法。

3）缺陷面积大小会对检测难度带来影响, 缺陷 面积越小, 最佳检测时间域越短。

\section{参考文献:}

[1] 赵艳, 葛新权, 李晓非. 城市生活垃圾产生量的影响因素分析[J]. 统 计与决策, 2016, 23: 91-94.

ZHAO Y, GE X Q, LI X F. Analysis of factors affecting the production of municipal domestic waste[J]. Statistics \& Decision, 2016, 23: 91-94.

[2] 孟小燕, 王毅, 苏利阳, 等. 我国普遍推行垃圾分类制度面临的问题 与对策分析 [J]. 生态经济, 2019, 35(5): 184-188.

MENG Xiaoyan, WANG Yi, SU Liyang, et al. Research on problems and countermeasures of the implementation of household solid waste separation system in China[J]. Ecological Economy, 2019, 35(5): 184-188.

[3] 谭爽. 城市生活垃圾分类政社合作的影响因素与多元路径一一基于 模糊集定性比较分析 [J]. 中国地质大学学报: 社会科学版, 2019, 19(2): 85-98.

TAN Shuang. Influencing factors and multi-path of cooperation between governments and social organizations in MSW sorting-- a fuzzy set qualitative comparison analysis(fsQCA)[J]. Journal of China University of Geosciences: Social Sciences Edition, 2019, 19(2): 85-98.

[4] 向锐, 雷国元, 徐亚, 等. 填埋场环境下 HDPE 膜老化特性及其对周 边地下水污染风险的影响[J]. 环境科学研究, 2019, 8(2): 1-12.

XIANG Rui, LEI Guoyuan, XU Ya, et al. Aging behaviors of HDPE gomembrane in landfill environment and its impact on pollution risk of surrounding groundwater[J]. Research of Environmental Science, 2019, 8(2): $1-12$
[5] Malekie S, Ziaie F, Feizi S, et al. Dosimetry characteristics of HDPE-SWCNT nanocomposite for real time application[J]. Nuclear Instruments and Methods in Physics Research Section A: Accelerators, Spectrometers, Detectors and Associated Equipment, 2016, 833: 127-133.

[6] 傅刚辉. HDPE 膜在垃圾处理中心的施工技术 [J]. 建筑技术, 2017, 48(11): 1208-1210.

FU Ganghui. Study on construction of HDPE geomembrane in garbage disposal center [J]. Architecture Technology, 2017, 48(11): 1208-1210.

[7] 能昌信, 孙新宇, 徐亚, 等. 垂直 HDPE 膜安装过程的电学破损检测 方法[J]. 环境工程学报, 2018, 12(1): 349-355.

NENG Changxin, SUN Xinyu, XU Ya, et al. Electrical method to locate damage in vertical HDPE geomembrane during installation[J]. Chinese Journal of Environmental Engineering, 2018, 12(1): 349-355.

[8] 牛奕, 马云, 李明明, 等. 非金属材料红外无损检测的建模和数值分 析[J]. 红外技术, 2019, 41(3): 214-219.

NIU Yi, MA Yun, LI Mingming, et al. Modeling and numerical analysis of infrared nondestructive testing of non-metallic materials[J]. Infrared Technology, 2019, 41(3): 214-219.

[9] 郑凯, 江海军, 陈力. 红外热波无损检测技术的研究现状与进展 [J]. 红外技术, 2018, 40(5): 401-411.

ZHENG Kai, JIANG Haijun, CHEN Li, Infrared thermography NDT and its development[J]. Infrared Technology, 2018, 40(5): 401-411.

[10] 高晓进, 周金帅, 江柏红, 等. C/SiC 复合材料的红外热像无损检测 研究[J]. 激光与红外, 2018, 48(6): 720-725.

GAO Xiaojin, ZHOU Jinshuai, JIANG Baihong. Research on infrared thermography nondestructive testing of C/Si C composite[J]. Laser \& Infrared, 2018, 48(6): 720-725.

[11] Chrysafi A P, Athanasopoulos N, Siakavellas N J. Damage detection on composite materials with active thermography and digital image processing[J]. International Journal of Thermal Sciences, 2017, 116: 242-253.

[12] 林隆荣, 钟舜聪, 伏喜斌, 等. 复合材料缺陷的脉冲热成像有限元 模拟研究 $[\mathrm{J}]$. 机电工程, 2016, 33(1): 18-23.

LIN Longrong, ZHONG Shuncong, FU Xinbin. Finite element analysis of pulsed infrared imaging of defects in composites[J]. Journal of Mechanical \& Electrical Engineering, 2016, 33(1): 18-23.

[13] 张剑, 齐暑华. 红外热成像技术在复合材料无损检测中的应用现状 [J]. 工程塑料应用, 2015, 43(11): 122-126.

ZHANG Jian, QI Shuhua. Application of infrared thermography in nondestructive testing of composites[J]. Engineering Plastics Application, 2015, 43(11): 122-126. 